\title{
An efficient pseudo-inverse solution to the inverse kinematic problem for six-joint manipulators
}

\author{
STEFANO CHIAVERINI $\dagger$ and OLAV EGELAND $\ddagger$
}

Keywords: Robotics, inverse kinematics, singularities.

\begin{abstract}
The use of the pseudo-inverse Jacobian matrix makes the solution of the inverse kinematic problem well-defined even at singular configurations of the robot arm; in the neighbourhood of a singularity, however, the computed solution often results in high joint velocities which may not be feasible to the real manipulator. Furthermore, the pseudo-inverse solution is computationally expensive, thus preventing real-time applications.

In this paper a systematic and efficient procedure to compute the pseudo inverse of the Jacobian matrix is presented which takes advantage of the kinematic analysis of the manipulator structure. In order to avoid excessive joint velocities close to singularities, the manipulator is treated as singular in a suitably defined region around each singularity. Inside this region a continuous joint velocity solution is achieved by interpolation in the dependent directions. The method is applied to a PUMA-like manipulator in simulations.
\end{abstract}

\section{Introduction}

The handling of singularities is one of the main problems in manipulator control. In a singular configuration the end effector loses degrees of freedom, and the inverse kinematic problem becomes overspecified.

Inverse kinematic solutions have traditionally been based on closed-form position transformations, or on the use of the inverse Jacobian to transform velocities or differential end-effector motion. These solution methods break down near singular configurations, and this is experienced in the form of very high joint velocities and large control deviations.

Singularities occur in the interior of the reachable workspace for typical six joint manipulators unless the workspace is restricted by joint limits. This means that the space where the end effector may be controlled in all directions will be a subset of the workspace. The singularity associated with spherical wrists is especially problematic as it is naturally characterized in wrist coordinates, but it can occur practically everywhere in the reachable workspace; so a six joint manipulator may have five or less degrees of freedom in significant parts of the workspace.

The use of redundant degrees of freedom is an interesting approach to handle the problem of singularities (Whitney 1972). If an extra joint is added in the shoulder of a PUMA-like manipulator, the end effector can have six degrees of freedom everywhere in the workspace (Egeland 1989, Hollerbach 1984). However, the redundant manipu-

Received 1 July 1990.

† Dipartimento di Informatica e Sistemistica, Università degli Studî di Napoli "Federico II", I-80125 Napoli, Italy.

$\ddagger$ Division of Engineering Cybernetics, Norwegian Institute of Technology, N-7034 Trondheim, Norway. 
lator will still have singular configurations which must be avoided or handled in the inverse kinematic algorithm.

The problem of singularities has been solved by planning trajectories that avoid singular configurations. Joint space interpolation has been proposed (Taylor 1979) when the planned trajectory is close to a singularity. In this case large control deviations in end-effector coordinates may result.

Balestrino, De Maria and Sciavicco (1984) and Wolovich and Elliott (1984) independently proposed the use of closed-loop inverse kinematic schemes based on the use of the Jacobian transpose in lieu of the Jacobian inverse. These methods do not converge quite as fast as inverse-based methods, but they work well also in singular configurations. The Jacobian transpose schemes have been further developed by Sciavicco and Siciliano (1988) and Das, Slotine and Sheridan (1988) to solve the inverse kinematic problem for redundant manipulators, and by Chiacchio and Siciliano (1988) to improve the performance close to singular configurations. The main problem here is to tune the algorithm for both robustness and high accuracy.

Wampler (1986) and Nakamura and Hanafusa (1986) used damped least-squares methods to obtain a modified Jacobian that was nonsingular in the whole workspace, and an approximate inverse kinematic solution was found. The problem here is to select suitable values for the damping parameters. High damping factors give good behaviour but reduced accuracy in the neighbourhood of singular points.

Whitney (1972) proposed to use nonsingular blocks of the Jacobian matrix to calculate an approximate solution at singular configurations. Aboaf and Paul (1987) studied the wrist singularity, and observed that the commanded angular velocity around the degenerate axis could not be achieved with finite joint velocities. The proposed solution was to eliminate this component from the reference velocity, and to specify one extra reference in joint space. Also Khatib (1987) proposed to eliminate commanded motion in degenerate directions, and demonstrated this for a three-link planar manipulator. The manipulator could then be regarded as a redundant manipulator in the subspace orthogonal to the degenerate direction. This makes it possible to apply techniques from redundant manipulator theory (Baillieul, Hollerbach and Brockett 1984, Egeland 1987, Khatib 1987, Nakamura, Hanafusa and Yoshikawa 1987, Sciavicco and Siciliano 1988, Wampler 1987). The main problem with this type of solution is to specify the degenerate directions in a systematic way, and to have smooth transitions between the usual inverse kinematic algorithm and the algorithms used close to the singularities.

In this paper a new solution to the problem of singularities in inverse kinematics recently proposed in Chiaverini and Egeland (1990) is illustrated in full. The method can be used both in off-line planning and in real-time sensory control. The different singularities of a particular manipulator are found and described as hypersurfaces in joint space. The corresponding degenerate directions are described in link-fixed frames. To avoid excessive joint velocities in the neighbourhood of the singular configurations, appropriate regions are defined around each singularity where the manipulator is treated as singular. Then an exact inverse kinematic solution is found in the space of feasible end-effector motion through the use of a generalized inverse of the manipulator Jacobian. This solution has the properties of minimum error in end-effector coordinates and minimum norm is joint space. Interpolation is used in the degenerate directions to achieve a continuous solution when the manipulator enters or leaves the region. This interpolation is in the nullspace of the Jacobian. The method is applied to a six-joint manipulator in simulations. 


\section{Basic concepts}

The direct kinematic equation describes the mapping of the $n$-dimensional joint coordinate vector $\boldsymbol{q}$ into the $m$-dimensional task-space coordinate vector $\boldsymbol{p}$ as

$$
p=f_{p}(q)
$$

where $f_{p}$ is a continuous nonlinear vector function.

The mapping between the joint velocity vector $\dot{\boldsymbol{q}}$ and the task-space velocity vector $\dot{p}$ can be obtained differentiating eqn. (1) with respect to time, yielding

$$
\dot{\boldsymbol{p}}=\boldsymbol{J}_{p}(\boldsymbol{q}) \dot{\boldsymbol{q}},
$$

where $J_{p}(q)$ is the $m \times n$ task Jacobian matrix associated with $\boldsymbol{f}_{p}(q)$. If for some $q_{s}$ the task Jacobian matrix happens to be rank deficient, the corresponding configuration is said to be singular.

Eqns. (1) and (2) constitute the direct kinematics of an assigned manipulator.

Since the manipulator task is usually defined by specifying a task-space trajectory, it is desirable to reconstruct the corresponding joint-space trajectory in order to apply control in joint coordinates. The computation of suitable mappings of the task-space variables into the joint-space variables constitutes the inverse kinematic problem.

When $n>m$ the manipulator is said to be redundant. In this case infinitely many joint solutions corresponding to the same task-space vector can be determined. In a singular configuration the end effector loses mobility in spite of the extra degrees of freedom available. Singularity avoidance can be achieved by using the internal motion to obtain a different joint solution; however, singular configurations will still be present requiring special handling in the solution of the inverse kinematic problem.

When $n=m$ the manipulator is said to be nonredundant. In this case the condition defining singular configurations can be stated more simply as $\operatorname{det} J_{p}\left(q_{s}\right)=0$; moreover, the inverse kinematic problem has a finite number of solutions almost everywhere in the task space.

The case $n<m$ has no practical interest, since the manipulator would be unable to provide all the degrees of freedom required by the task.

Throughout the paper the case $m=6$ will be considered. The task-space vector $p$ is defined so that the first three components are the Cartesian coordinates of the end effector, and the last three a suitable minimal representation of end-effector orientation.

When a three-parameter description of orientation (such as Euler angles or RollPitch-Yaw representation) is used as in $p$, there will always be mathematical singularities where the mapping (2) is singular although the end effector has six degrees of freedom.

Description without mathematical singularities are based on end-effector velocity or on differential motion. The end-effector velocity vector $v$ is defined as

$$
v=\left(\begin{array}{c}
\boldsymbol{v}_{\boldsymbol{t}} \\
\omega
\end{array}\right),
$$

where $v_{t}$ is the three-dimensional vector of translational velocities in base coordinates and $\omega$ is the three-dimensional vector of angular velocities in base coordinates. The mapping

$$
\boldsymbol{v}=\boldsymbol{J}(\boldsymbol{q}) \dot{\boldsymbol{q}}
$$

will be singular only in configurations corresponding to actual mechanical singularities; rank deficiencies in the $6 \times n$ matrix $J$, indeed, imply a loss of mobility related to 
some subspace of the end-effector velocity space which cannot be spanned in that configuration. The matrix $\boldsymbol{J}$ is termed the base Jacobian or simply the Jacobian of the manipulator.

The relation between the base Jacobian $\boldsymbol{J}$ and the task Jacobian $\boldsymbol{J}_{\boldsymbol{p}}$ is given by

$$
J_{p}(q)=J_{p v}(p) J(q),
$$

where $J_{p v}(p)$ is a $6 \times 6$ transformation matrix that is a function only of $p$. The singularities of the $\boldsymbol{J}_{p v}$ matrix are the mathematical singularities of the mapping (2).

To overcome the problems related to the use of the $\boldsymbol{p}$ vector, an alternative possibility of minimal description can be exploited. It consists in using different minimal descriptions of orientations in different regions of the manipulator task space, so that each description be singularity-frec inside the corresponding region. However, problems arise when the assigned trajectory crosses a region boundary which in turn implies a discontinuity in the representation of orientation.

In the following, it is assumed that a coordinate transformation matrix is used to describe absolute rotation, while velocity and differential end-effector motion is described in terms of $\boldsymbol{v}$ and $\delta \boldsymbol{x}=\boldsymbol{v} \delta t$. We will therefore mainly be working on the mapping (4) and

$$
\delta \boldsymbol{x}=\boldsymbol{J}(\boldsymbol{q}) \delta \boldsymbol{q},
$$

where $\delta \boldsymbol{q}=\dot{\boldsymbol{q}} \delta t$ is the vector of differential joint motions.

It can be seen (Nakamura and Hanafusa 1986) that the inverse kinematic problem in position, velocity or acceleration can be solved using linear equations with the Jacobian matrix as the coefficient matrix. Therefore, eqn. (6) will be used to discuss the inverse kinematic problem without loss of generality.

\section{Problem statement}

In the following the inverse kinematic problem for nonredundant manipulators will be discussed.

The most direct approach to resolve differential joint motions $\delta q$ corresponding to the differential end-effector motion $\delta \boldsymbol{x}$ is based on the use of the inverse Jacobian matrix as follows

$$
\delta \boldsymbol{q}=\boldsymbol{J}^{-1}(\boldsymbol{q}) \delta \boldsymbol{x} .
$$

This solution presents two major limitations:

(a) $J^{-1}(q)$ is not defined at a singular configuration; this implies that it is not possible to determine a joint motion $\delta q$ corresponding to an assigned $\delta \boldsymbol{x}$;

(b) in the neighbourhood of a singular configuration eqn. (7) gives an exact solution that often results in high joint velocities representing an infeasible solution for the manipulator.

As a better approach to the inverse kinematic solution, the use of generalized inverses of the Jacobian matrix has been proposed. It must be noticed here that several techniques presented in the literature and consisting in removing end-effector reference components (Aboaf and Paul 1987, Khatib 1987) or in using nonsingular blocks of the Jacobian matrix (Whitney 1972) can be in some way arranged in this kind of approach. 
We have worked with the pseudo inverse of the Jacobian. The pseudo inverse $\boldsymbol{J}^{\dagger}$ of $\boldsymbol{J}$ is a unique matrix which satisfies the conditions in Zadeh and Desoer (1963).

$$
\begin{aligned}
\boldsymbol{J}^{\dagger} \boldsymbol{J}_{\boldsymbol{u}}=\boldsymbol{u} & \forall \boldsymbol{u} \in \mathscr{N}(\boldsymbol{J})^{\perp}=\mathscr{R}\left(\boldsymbol{J}^{\mathbf{T}}\right) \\
\boldsymbol{J}^{\dagger} \boldsymbol{v}=\boldsymbol{0} & \forall \boldsymbol{v} \in \mathscr{R}(\boldsymbol{J})^{\perp}=\mathscr{N}\left(\boldsymbol{J}^{\mathbf{T}}\right) \\
\boldsymbol{J}^{\dagger}(\boldsymbol{v}+\boldsymbol{w})=\boldsymbol{J}^{\dagger} \boldsymbol{v}+\boldsymbol{J}^{\dagger} \boldsymbol{w} & \forall \boldsymbol{v} \in \mathscr{R}(\boldsymbol{J}) \quad \forall \boldsymbol{w} \in \mathscr{R}(\boldsymbol{J})^{\perp} .
\end{aligned}
$$

The pseudo inverse can alternatively be defined with the equations in Ben-Israel and Greville (1974).

$$
\begin{aligned}
\boldsymbol{J}^{\dagger} \boldsymbol{J} & =\boldsymbol{J} \\
\boldsymbol{J}^{\dagger} \boldsymbol{J} \boldsymbol{J}^{\dagger} & =\boldsymbol{J}^{\dagger} \\
\left(\boldsymbol{J} \boldsymbol{J}^{\dagger}\right)^{\mathbf{T}} & =\boldsymbol{J}^{\dagger} \\
\left(\boldsymbol{J}^{\dagger} \boldsymbol{J}^{\mathbf{T}}\right. & =\boldsymbol{J}^{\dagger} \boldsymbol{J} .
\end{aligned}
$$

The pseudo inverse has the property that the $\delta q$ given by

$$
\delta \boldsymbol{q}=\boldsymbol{J}^{+} \delta \boldsymbol{x}
$$

is the least-squares solution with minimum norm for eqn. (6); that is it the solution satisfying

$$
\min _{\delta \boldsymbol{q}}\|\delta q\|
$$

of all the $\delta q$ that fulfill the equation

$$
\min _{\delta \boldsymbol{q}}\|\delta \boldsymbol{x}-\boldsymbol{J}(\boldsymbol{q}) \delta \boldsymbol{q}\|,
$$

where $\|\cdot\|$ denotes the Euclidean norm.

Although solution (15) is defined even for singular configurations, high joint velocities will still result in the neighbourhood of singularities. This happens because eqn. (15) is equivalent to eqn. (7) at nonsingular configurations, while it discontinuously offers an approximate solution at singularities. Moreover, this latter aspect is source of additional problems since it implies discontinuous solutions in joint space.

In order to exploit the potential of solution (15) two main problems remain unsolved:

(a) a systematic and efficient procedure to compute the pseudo inverse of the Jacobian matrix is needed;

(b) the continuity of the joint space solution must be ensured.

A solution to these problems is presented in this paper.

\section{Computation of pseudo-inverse Jacobian}

In the following a systematic framework to compute the pseudo inverse is developed based on a modification of an algorithm proposed by Mayne (1969). The algorithm at issuc consists of the following steps:

(1) given an $m \times n$ matrix $\boldsymbol{J}$ with $m \leqslant n$ and $\operatorname{rank}(\boldsymbol{J})=r \leqslant m$, by means of row operations (equivalent to premultiplication by an $m \times m$ matrix $P$ ) reduce $J$ to the matrix

$$
\left(\begin{array}{l}
J_{1} \\
J_{2}
\end{array}\right)
$$


where $J_{1}$ is an $r \times n$ matrix of full rank, and $J_{2}$ is an $(m-r) \times n$ matrix ${ }^{1}$;

(2) The pseudo inverse of the matrix $J$ is given by

$$
J^{\dagger}=J_{1}^{\mathrm{T}}\left(\boldsymbol{G} G^{\mathrm{T}}\right)^{-1} \boldsymbol{G},
$$

where the $r \times m$ matrix $G$ is computed as follows

$$
\boldsymbol{G}=\boldsymbol{J}_{1} \boldsymbol{J}^{\mathbf{T}} \text {. }
$$

The $J^{\dagger}$ matrix given by (19) and (20) satisfies the conditions (8), (9) and (10) and is the pseudo inverse of $\boldsymbol{J}$.

This can be shown as in Mayne (1969) by first noting that $\boldsymbol{G G}^{\mathrm{T}}$ has rank $r$ and is invertible as the columns of $\boldsymbol{G}^{\mathrm{T}}=\boldsymbol{J J}_{1}^{\mathrm{T}}$ span the range of $\boldsymbol{J}$ which is of dimension $\boldsymbol{r}$.

If $\boldsymbol{u \in \mathscr { R }}\left(\boldsymbol{J}^{\mathrm{T}}\right)$ then $\boldsymbol{u}=\boldsymbol{J}_{1}^{\mathrm{T}} \boldsymbol{a}$ for some $r$-dimensional vector $\boldsymbol{a}$. Then

$$
\boldsymbol{J}^{\dagger} \boldsymbol{J} \boldsymbol{u}=\boldsymbol{J}_{1}^{\mathrm{T}}\left(\boldsymbol{G} G^{\mathrm{T}}\right)^{-1} \boldsymbol{G} \boldsymbol{J} \boldsymbol{J}_{1}^{\mathrm{T}} \boldsymbol{a}=\boldsymbol{u}
$$

which means that $(8)$ is satisfied.

If $\boldsymbol{v} \in \mathcal{N}\left(\boldsymbol{J}^{\mathrm{T}}\right)$ then $\boldsymbol{J}^{\mathrm{T}} \boldsymbol{v}=\mathbf{0}$. Then

$$
J^{\dagger} v=J_{1}^{T}\left(G G^{T}\right)^{-1} J_{1} J v=0,
$$

so (9) is also satisfied. Finally, the condition (10) is trivially true for $\mathbf{J}^{\dagger}$.

It is clear that the main problem with this algorithm is to select the matrix $P$ used in the generation of $J_{1}$. In the remainder a new systematic approach to derive a suitable matrix $\boldsymbol{P}$ is presented. To this end we firstly introduce the concept of feasible motion and dependent end-effector directions.

Let $\mathscr{R}(\boldsymbol{J}(\hat{\boldsymbol{q}}))$ be the range space of the matrix $\boldsymbol{J}$ at the $\hat{\boldsymbol{q}}$ configuration. If $\hat{\boldsymbol{q}}$ is nonsingular, then

$$
\operatorname{dim}[\mathscr{R}(\boldsymbol{J}(\hat{q}))]=m
$$

When $\hat{\boldsymbol{q}}$ is singular, instead,

$$
\operatorname{dim}[\mathscr{R}(\boldsymbol{J}(\hat{\boldsymbol{q}}))]=r \quad r<m .
$$

$\mathscr{R}(\boldsymbol{J}(\hat{\boldsymbol{q}}))$ is the subspace of feasible motion in the $m$-dimensional space of end-effector differential motion at the $\hat{\boldsymbol{q}}$ configuration. Equation (24) states that this subspace has a dimension $r$ less than $m$ at a singular configuration. This means that a differential endeffector motion can be specified arbitrarily only in $r$ directions; the motion in the remaining $m-r$ directions is determined dependently. These directions are termed the dependent directions.

Assume that a six-dimensional base is found where the $r$ first base vectors span $\mathscr{R}(\boldsymbol{J})$. If a feasible end-effector motion $\delta \boldsymbol{x}$ is transformed to this base, it will have components

$$
\left(\begin{array}{l}
\delta x_{1} \\
\delta x_{2}
\end{array}\right)
$$

where $\delta x_{1} \in R^{r}$ and $\delta x_{2} \in R^{m-r}$. The components of $\delta x_{1}$ are independent, while the components in $\delta x_{2}$ depends on $\delta x_{1}$.

Consider now the equation defining the matrix (18):

$$
\boldsymbol{P J}=\left(\begin{array}{c}
\boldsymbol{J}_{1} \\
\boldsymbol{J}_{2}
\end{array}\right)
$$

\footnotetext{
'In Mayne's algorithm (1969) $J_{2}$ was the zero matrix.
} 
postmultiplying by $\delta q$ gives

$$
\boldsymbol{P} \delta \boldsymbol{x}=\left(\begin{array}{l}
\delta \tilde{\boldsymbol{x}}_{1} \\
\delta \tilde{\boldsymbol{x}}_{2}
\end{array}\right)
$$

where $\delta \tilde{\boldsymbol{x}}_{1} \in \boldsymbol{R}^{r}$ and $\delta \tilde{\boldsymbol{x}}_{2} \in \boldsymbol{R}^{m-\boldsymbol{r}}$. As the rank of $\boldsymbol{J}_{1}$ is the same as the rank of $\boldsymbol{J}$, it is clear that the right-hand-side vector has the same form as in (25) with $r$ independent components first and then $m-r$ dependent components. Since $\delta \boldsymbol{x} \in \mathscr{R}(\boldsymbol{J})$, we recognize that the matrix $\boldsymbol{P}$ is indeed a projector onto a base of the end-effector motion space where the first $r$ base vectors span the subspace of feasible motion, and the last $m-r$ vectors represent dependent directions.

The problem now is to build this special base as simply as possible.

\section{Description of the dependent directions}

For a particular manipulator it is usually possible to find classes of singular configurations consisting of all singularities for which the same mechanical origin can be determined. The wrist singularity, for instance, is actually a class of singular configurations rather than one singular configuration.

A class of singular configurations which can be determined according to the above concept will be termed a structural singularity. The structural singularities for a particular manipulator can be described in terms of hypersurfaces in joint space. We assume that a given manipulator has a finite and small number of structural singularities. This might not be true for all possible kinematic designs, but we are only interested in good designs with few singularities.

Just as the singularities are described in joint space, the dependent axes can be described in terms of axes that are fixed in some link cordinate system. The physical interpretation of this is that the end-effector can be given full mobility in a singularity by suitably including $m-r$ extra joints in the arm. In fact for common industrial manipulators the degenerate directions can be selected along axes in the coordinate systems specified by the Denavit-Hartenberg convention, and this makes it possible to find the projection matrix $\boldsymbol{P}$ in eqn. (26) with very little extra computation.

To justify this claim a six-joint manipulator with the conventional design of three inner joints for positioning and three outer rotary joints for end-effector orientation is investigated.

Two types of dependent axes may appear in singular configurations.

The end effector may lose one rotational degree of freedom around some axis. Surely this degenerate axis must be orthogonal to the rotational axis of joint six. If the manipulator has the usual design where joint axes five and six are orthogonal it is clear that the degenerate axis is also orthogonal to the rotational axis of joint five. Then, according to the Denavit-Hartenberg convention the dependent axis must $x_{5}$ which is the $x$-axis in coordinate frame five. This singularity is termed the wrist singularity. Note that there might be a rotation around an axis parallel to the dependent axis; the degeneracy is that this rotation is dependent on the $r$ independent components of the feasible motion.

The end effector may also lose translational freedom along one or two axes. This may occur in two situations. The first is when there are two or three neighbouring pitch joints with parallel axes and all the pitch joints except the first are stretched out. This singularity is termed the elbow singularity. The dependent axis is in the direction of the offset between the joints, and this is an $x$-axis in the Denavit-Hartenberg convention. 
The second situation is when a roll joint which is supposed to provide a translation has a zero arm. This is normally only possible for joint 1 , and it occurs when the wrist point is on joint axis 1 . This singularlity is termed the shoulder singularity. The dependent axis of the shoulder singularity will be in the direction of the axis joint 2 for the architecture of the PUMA and Cincinnati T3, and this is the $z_{2}$-axis according to the Denavit-Hartenberg formalism.

In the following we will assume that the dependent axes are along some axis in the Denavit-Hatenberg frames. The extension to cases where the dependent axis is along some other link-fixed axis is straightforward.

Let $\hat{\boldsymbol{q}}$ be a singular configuration for which a dependent axis is described in the coordinate frame $i$ fixed in link $i$. Assume that the degeneracy is a dependent velocity along one of the axes of frame $i$, and let $C_{0}^{i}(\hat{q})$ be the $3 \times 3$ coordinate transformation matrix which transforms a vector decomposed in frame 0 to frame $i$. The end-effector velocity decomposed in frame $i$

$$
\boldsymbol{v}_{\boldsymbol{t}}^{i}=\boldsymbol{C}_{\mathrm{o}}^{i}(\hat{\boldsymbol{q}}) \boldsymbol{v}_{\boldsymbol{t}}
$$

will then have one component which is dependent for all feasible end-effector velocities $v_{t}$. This means that the projection matrix

$$
\tilde{\boldsymbol{P}}=\left(\begin{array}{cc}
\boldsymbol{C}_{0}^{i}(\hat{\boldsymbol{q}}) & \mathbf{0} \\
\mathbf{0} & \boldsymbol{I}
\end{array}\right)
$$

can be used. The permutation matrix $\boldsymbol{P}$ in eqn. (26) is obtained by exchanging the dependent row with the last row in $\tilde{\boldsymbol{P}}$.

If the degeneracy is in the form of a dependent rotation around an axis in the frame $j$ fixed in link $j$, the angular velocity decomposed in frame $j$

$$
\omega^{j}=\boldsymbol{C}_{0}^{j}(\hat{q}) \omega
$$

has one component which is dependent for all feasible end-effector rotations. Here, $\boldsymbol{C}_{0}^{j}(\hat{\boldsymbol{q}})$ is the $3 \times 3$ coordinate transformation matrix from frame 0 to frame $j$. The projection matrix

$$
\tilde{\boldsymbol{P}}=\left(\begin{array}{cc}
I & 0 \\
0 & C_{0}^{j}(\hat{q})
\end{array}\right)
$$

can then be used; as above the matrix $\boldsymbol{P}$ is found by exchanging the dependent row in $\tilde{\boldsymbol{P}}$ with the last row.

The application to multiple singularities of this type is straightforward. With a degenerate translation along an axis in frame $i$ and a dependent rotation around an axis in frame $j$ the projection matrix is simply

$$
\tilde{\boldsymbol{P}}=\left(\begin{array}{cc}
C_{0}^{i}(\hat{\boldsymbol{q}}) & 0 \\
\mathbf{0} & \boldsymbol{C}_{0}^{j}(\hat{\boldsymbol{q}})
\end{array}\right)
$$

With two dependent translations along axes in frames $i$ and $j$, the solution is more complicated if the dependent axes are not orthogonal. However, in a six-joint manipulator with vertical first axis and horizontal and parallel second and third axes, the two possible translational degeneracies are the shoulder and elbow singularities, and these have orthogonal dependent axes. Then the projection matrix $\tilde{\boldsymbol{P}}$ is as with a single translational degeneracy. 
It must be noticed here that, since we are working on structural singularities, the structure of the projection matrices can be found once and for all for a given manipulator as there are only a limited number of different cases.

\section{Restriction of motion close to singular configurations}

Close to a singular configuration the inverse kinematic problem becomes illconditioned as a small change in end-effector position may give a very large change in joint position. To solve this problem and to ensure a continuous solution when the trajectory goes through a singularity, a region is introduced around the singular configuration where the manipulator is treated as if it were singular. This region is termed the restricted region.

The pseudo inverse of the Jacobian is calculated using eqns. (19) and (20). Assume that the rank of the Jacobian is $\hat{r}$ in a singular configuration $\hat{q}$. Then $J_{1}(\hat{q})$ has dimension $\hat{r} \times m$. According to the concept of restricted regions the $\hat{r} \times m$ matrix $J_{1}(q)$ is used in the computation of the pseudo inverse in the whole restricted region and not only in the singularity. As a result of this, the computed pseudo inverse becomes discontinuous at the border of the restricted region instead of at the singularity.

The computed pseudo inverse is denoted $\mathrm{J}^{-}$and is defined by

$$
\begin{gathered}
\boldsymbol{J}^{-}=\tilde{\boldsymbol{J}}_{1}^{\mathrm{T}}\left(\boldsymbol{G} \boldsymbol{G}^{\mathrm{T}}\right)^{-1} \boldsymbol{G} \\
\boldsymbol{G}=\tilde{\boldsymbol{J}}_{1} \boldsymbol{J}^{\mathrm{T}}
\end{gathered}
$$

where $\tilde{J}_{1}$ has the same structure in the whole restricted region.

In the case where the dependent axes are orthogonal and fixed in DenavitHartenberg frames it is simple to specify the restricted regions. The size of the regions should be selected so that the end effector can achieve an assigned velocity in all directions outside the restricted region.

The singularities of the PUMA-type kinematic structure are used in the following examples to demonstrate how the regions can be specified.

First the wrist is studied. The singularity occurs when the first and the third wrist axes are colinear. This condition is satisfied by all the configurations belonging to the joint-space hypersurface

$$
q_{5}=0
$$

The $x_{5}$-axis is selected as the dependent direction. The angular velocity of the end effector in frame 5 coordinates is

$$
\omega^{5}=\left(\omega_{x_{s}} \omega_{y_{5}} \omega_{z y}\right)^{\mathrm{T}}
$$

The angular velocity around the $x_{5}$-axis is

$$
\omega_{x_{5}}=\dot{q}_{4} \sin \left(q_{5}\right) \text {. }
$$

The restricted region is found as the region where the end effector cannot achieve a given $\hat{\omega}_{x_{5}}$ with $\left|\dot{q}_{4}\right| \leqslant \dot{q}_{4}^{\max }$; it is given by

$$
\left|q_{5}\right| \leqslant \arcsin \left(\hat{\omega}_{x_{5}} / \dot{q}_{4}^{\max }\right)
$$

where the region boundaries are computed only once.

The second singularity is a pitch joint singularity; it is described by the hypersurface

$$
q_{3}=0 .
$$


The degeneracy is a translation in the $x_{2}$ direction which cannot be independently specified. The end-effector translational velocity in frame 2 coordinates is

$$
v_{t}^{2}=\left(v_{x_{2}} v_{y_{2}} v_{z_{2}}\right)^{\mathrm{T}} \text {. }
$$

The velocity in the $x_{2}$ direction from joint 3 is

$$
v_{x_{2}}=-\dot{q}_{3} d_{4} \sin \left(q_{3}\right) \text {, }
$$

where $d_{4}$ is the length of link 3 . The restricted region is in this case given by

$$
\left.\left|q_{3}\right| \leqslant \arcsin \left(\hat{v}_{x_{2}} / \dot{q}_{3}^{\max } d_{4}\right)\right)
$$

if it is specified that the end effector must be able to achieve an assigned $\hat{v}_{x_{2}}$ outside the restricted region with $\left|\dot{q}_{3}\right| \leqslant \dot{q}_{3}^{\max }$.

Finally the roll joint singularity is investigated. The singularity in this case occurs when the wrist point is on the rotational axis of joint 1 ; the corresponding joint-space hypersurface is given by

$$
r_{w}=a_{2} \sin \left(q_{2}\right)+d_{4} \sin \left(q_{2}+q_{3}\right)=0,
$$

where $a_{2}$ is the length of link 2 and $d_{4}$ is the length of link 3 . The dependent axis is the $z_{2}$ axis where the translation depends on the orientation of the end effector. Note that this dependent axis is orthogonal to the dependent $x_{2}$ axis studied above. The velocity in the $z_{2}$ direction is

$$
v_{z_{2}}=r_{w} \dot{q}_{1}
$$

and the restricted region can be calculated from

$$
\left|r_{w}\right| \leqslant \hat{v}_{z_{2}} / \dot{q}_{1}^{\max },
$$

where $\hat{v}_{z_{2}}$ again is the velocity requirement outside the restricted region.

\section{Nullspace motion and continuity of the joint-space solution}

The manipulator is treated as singular in the restricted regions, which means that the commanded task space motion is only achieved for $r$ degrees of freedom. The remaining $n-r$ degrees of freedom must be commanded so that the solution is continuous when the manipulator enters or leaves the region. These degrees of freedom are in the nullspace of the manipulator Jacobian.

The obvious solution to this problem is to interpolate between the singularity and a point on the border of the restricted region termed the border point and which has coordinates $q_{b}$. This is done in our scheme, however we would like to emphasise that the interpolation is only performed in the dependent directions, and that an exact solution is used in the space of feasible end-effector motion. The scheme resembles the solution by Aboaf and Paul (1987). However in their work joint 4 was given maximum velocity in the restricted region associated with the wrist singularity, and this may result in a discontinuous velocity in joint 4 due to a change of sign when the manipulator leaves the restricted region.

The differential motion in joint space is found from

$$
\delta q=J^{-}(q) \delta x+\left(I-J^{-}(q) J(q)\right) z,
$$

where $\left(\boldsymbol{I}-\boldsymbol{J}^{-} \boldsymbol{J}\right)$ projects an arbitrary vector $z$ into $\mathcal{N}\left(\widetilde{J}_{1}\right)$ which is identical to the nullspace of $\boldsymbol{J}$ in the singularity. 
We first assume that the manipulator enters the restricted region of a singularity of multiplicity one, that is $r=m-1$. A continuous solution is then obtained by using

$$
z=\alpha J^{-1}\left(q_{b}\right) \delta x,
$$

where $\alpha$ is the interpolation factor which is zero in the singularity and unity on the border.

In the case of multiple singularities, one interpolation factor has to be used for each singularity to ensure continuity when the manipulator comes from one restricted region to another region associated with a singularity of a different dimension. A continuous solution could now be obtained using multiple border points, but this gives a complex solution with much computation.

We therefore chose to interpolate only the dependent motion associated with the wrist singularity in the case of multiple singularities. The wrist singularity is the most problematic as it can appear anywhere in the reachable workspace. A small discontinuity in the dependent motion associated with the shoulder and elbow can be tolerated, however it would also be possible to remove the discontinuity by low-pass filtering.

\section{Comparison with other approaches: an example}

In this section an example proposed by Wampler (1986) is revisited. The simple case considered is suitable for a comparison with other existing approaches.

Let the assigned manipulator consist of a single link of unit length with a rotational joint, and suppose that only the position of the endpoint along an horizontal line is the considered task-space coordinate $x$.

If $\vartheta$ is the angle measuring the rotation of the link from the horizontal line, the direct kinematics of the manipulator is given by

$$
\begin{aligned}
& x=\cos (\vartheta) \\
& \dot{x}=(-\sin (\vartheta)) \dot{\vartheta} \quad \vartheta \in]-\pi, \pi[.
\end{aligned}
$$

It is easily recognized that

$$
\boldsymbol{J}(\vartheta)=(-\sin (9))
$$

is the $1 \times 1$ Jacobian matrix of the assigned manipulator and $\vartheta=0$ is a singular configuration.

Inverse-kinematic velocity solution describes the mapping of the end-effector velocity space into the joint velocity space. In the simple monodimensional case the mapping just consists in a scaling factor depending on the position of the link; this scaling factor, defined as

$$
\rho(\hat{\vartheta})=\dot{\vartheta} / \dot{x},
$$

will be termed the velocity ratio in the $\Im$ configuration. In the following for simplicity of notation the dependence on the configuration will be dropped.

The inverse-based solution is given by

$$
\dot{\vartheta}=\left(-\frac{1}{\sin (\vartheta)}\right) \dot{x} ;
$$

it is not defined in $\vartheta=0$ and gives high velocity ratios as $\vartheta$ approaches the singularity. 
A solution defined even in the singular configuration is provided by a pseudo inverse of the Jacobian matrix. In this case it can be simply written as

$$
\dot{\vartheta}= \begin{cases}\left(-\frac{1}{\sin (\vartheta)}\right) \dot{x} & \vartheta \neq 0 \\ 0 & \vartheta=0 .\end{cases}
$$

High velocity ratios will still result in the neighbourhood of the singularity.

Bounded velocity ratios can be obtained modifying this latter solution. If a threshold value $\varepsilon$ is introduced, the joint velocity can be computed as

$$
\dot{\vartheta}= \begin{cases}\left(-\frac{1}{\sin (\vartheta)}\right) \dot{x} & |\sin (\vartheta)| \geqslant \varepsilon \\ 0 & |\sin (\vartheta)|<\varepsilon .\end{cases}
$$

For an allowed maximum velocity ratio $\rho_{\max }$ we choose $\varepsilon=1 / \rho_{\max }$

Although solution (53) has overcome our earlier problems, it does not constitute a feasible control strategy. Indeed, if the manipulator crosses the singular region, a discontinuous joint velocity results when the value of $\sin (9)$ imples a switch in the velocity law. Moreover, inside the singular region the degenerate direction cannot be controlled.

A more complete way of exploiting the generalized-inverse approach has been presented in this paper. The proposed solution, indeed, allows to achieve the desired continuous behaviour. In the case at issue the restricted region is defined by $|\sin (\vartheta)|<\varepsilon$ and the border point is $\vartheta_{b}=\arcsin (\varepsilon)$; choosing the interpolation factor $\alpha=\vartheta / \vartheta_{b}$, the solution is given by

$$
\dot{\vartheta}= \begin{cases}\left(-\frac{1}{\sin (\vartheta)}\right) \dot{x} & |\sin (\vartheta)| \geqslant \varepsilon \\ \left(-\frac{\vartheta}{\varepsilon \vartheta_{b}}\right) \dot{x} & |\sin (\vartheta)|<\varepsilon .\end{cases}
$$

A simple linear interpolation has been used here, whereas different possibilities can be exploited. The central point is that as long as the manipulator is treated as singular, what is lost in the trajectory achievement can be gained by fulfilling additional requirements. The basic requirement to ensure is the continuity of the velocity law, while a different shaping of the null-space term can be designed to meet different criteria.

The damped least-squares solution is

$$
\dot{\vartheta}=\left(-\frac{\sin (\vartheta)}{\sin ^{2}(\vartheta)+k^{2}}\right) \dot{x},
$$

where $k$ must be suitably chosen. For a direct comparison, we suppose that the same maximum velocity ratio $\rho_{\max }$ is assigned (see Fig. 1); it can be seen that this means $k=1 / 2 \rho_{\max }$.

Let us now compare the performance of the solutions (54) and (55).

Outside the singular region the proposed solution represents the exact solution. 


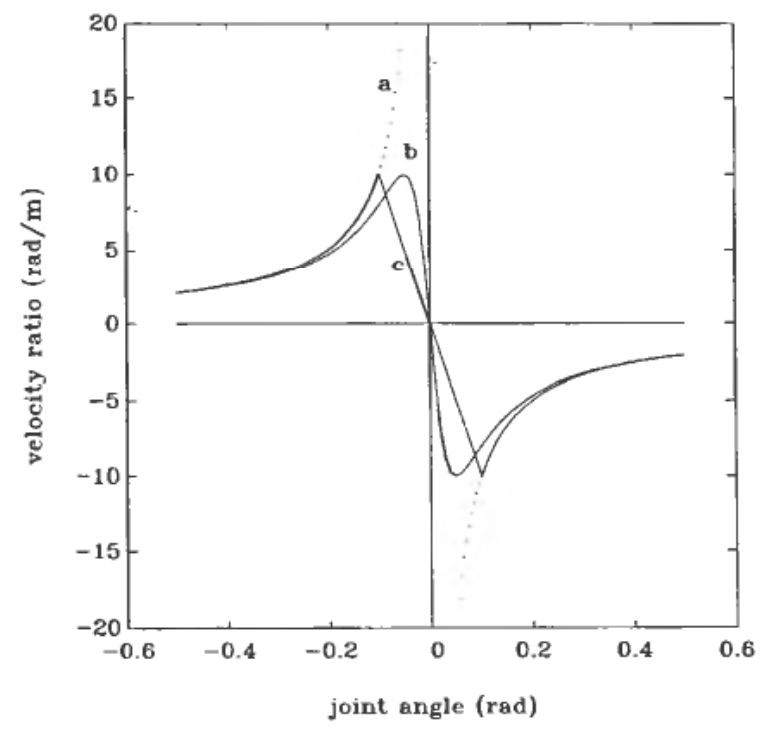

Figure 1. Direct compensation: a, exact solution; $b$, damped least-squares solution; $c$, proposed solution.

The damped least-squares method, instead, gives an error which takes its maximum value in correspondence of the $\vartheta_{b}$ angle; notice that with the assumed design this error is equal to the 20 per cent of the maximum velocity ratio. A different choice of $k$ allows for better tracking performance. If a maximum error equal to the 1 per cent of $\rho_{\max }$ is desired, a peak in the velocity ratio up to 5 times the assigned $\rho_{\max }$ will result. Moreover, a faster change in the velocity characteristic in the very neighbourhood of $\vartheta=0$ is required.

Inside the singular region, both approaches are based on modification of the exact transformation (51) in order to exchange precision with feasibility of the solution; quality rather than accuracy of the solution is therefore at issue. It can be seen that the damped least-squares solution (55) results in higher velocity ratios and in sharper transition of the velocity characteristic in the neighbourhood of the singularity, unless designs giving unacceptable errors in larger regions of the workspace are considered. On the other hand it must be noticed that the velocity characteristic for the proposed solution (54) is not fixed inside the restricted region; if it is convenient, a different nullspace term can be used instead of the linear one without affecting the performance of the solution far from the singularity.

A direct comparison with the Jacobian-transpose methods is not possible, since they are inherently iterative methods. This means that, instead of an instantaneous mapping of the end-effector velocity into the joint velocity, a tracking-error dependent joint velocity law is used. For the assigned manipulator the Jacobian-transpose solution is given by

$$
\dot{\vartheta}=\beta(\sin (\vartheta))\left[x_{d}-\cos (\vartheta)\right]
$$

where $\beta$ is a suitable gain and $x_{d}$ is the time-varying reference trajectory for the taskspace coordinate $x$. It must be noticed that the transpose solution is well defined also in the singular configuration. 


\section{Case studies and simulation results}

An industrial manipulator has been considered for practical implementation of the proposed algorithm. The manipulator is the MANUTEC_R3 robot possessing six joints arranged in a PUMA-like geometry (see Fig. 2).

The given kinematic structure presents the typical elbow, shoulder, and wrist singularities. According to Section 6, the restricted regions are specified as follows:

$$
\begin{array}{ll}
\left|q_{5}\right| \leqslant \arcsin (0.05)=\hat{q}_{b_{5}} & \text { (wrist) } \\
\left|q_{3}\right| \leqslant \arcsin (0.05)=\hat{q}_{b_{3}} & \text { (elbow) } \\
\left|r_{w}\right| \leqslant 0.05 & \text { (shoulder). }
\end{array}
$$

In the wrist and elbow cases, this choice means to assign an upper bound of 20 on the velocity ratio along the dependent direction; in the shoulder case, in particular, the restricted region can be visualized as a cylinder of $5 \mathrm{~cm}$ radius around the shoulder axis of the manipulator.

When the manipulator enters at least one of the above regions, it is treated as singular; this means that the solution (7) is switched to the solution $(46,47)$. To the purpose, some extra computation is needed.

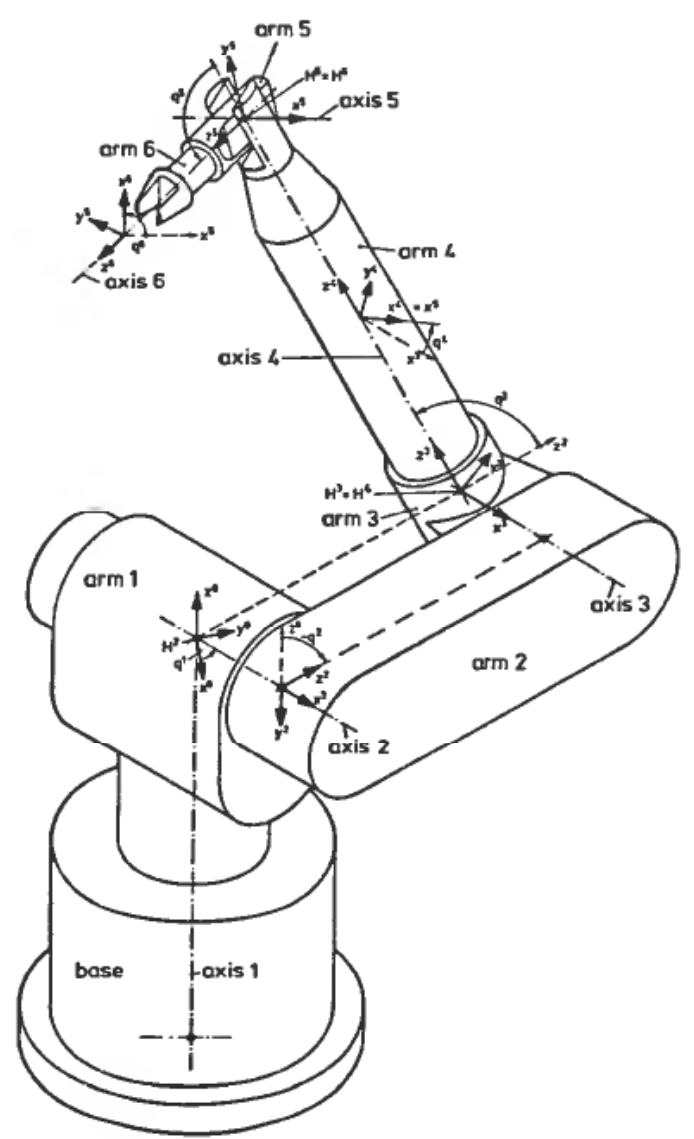

Figure 2. Model of the MANUTEC_R3 robot. 
First the matrix $\tilde{\boldsymbol{P}}$ is computed either as

$$
\left(\begin{array}{cc}
I & 0 \\
0 & C_{0}^{5}(q)
\end{array}\right)
$$

in the wrist singularity case alone, or as

$$
\left(\begin{array}{cc}
C_{0}^{2}(q) & 0 \\
0 & I
\end{array}\right)
$$

in the elbow and/or shoulder singularity case, or as

$$
\left(\begin{array}{cc}
C_{0}^{2}(q) & \mathbf{0} \\
0 & C_{0}^{5}(q)
\end{array}\right)
$$

in all other singular cases. Note that the matrices $\boldsymbol{C}_{0}^{i}(\boldsymbol{q})$ are already available since they are needed in the computation of the direct kinematic function.

Then the block $\boldsymbol{P}_{1}$ of the matrix $\boldsymbol{P}$ (needed to build the matrix $\boldsymbol{J}_{1}$ ) is obtained by removing the rows of the matrix $\tilde{\boldsymbol{P}}$ which correspond to dependent directions; namely the first, third, and the fourth row respectively in the case of elbow, shoulder, and wrist singularity. The proposed pseudo inverse will then be computed as in eqns. (33) and (34), since $\tilde{\boldsymbol{J}}=\boldsymbol{P}_{1} \boldsymbol{J}$.

The computation of the border point $\boldsymbol{q}_{b}$ is performed in successive steps. First it is assumed $\boldsymbol{q}_{b}=\boldsymbol{q}$; then in the wrist singularity case it is $q_{b_{5}}=\hat{q}_{b_{5}}$ and in the elbow singularity case it is $q_{b_{3}}=\hat{q}_{b_{3}}$. The shoulder singularity case is not trivial because of the nonlinear coupling between $q_{2}$ and $q_{3}$. Our choice is to search $q_{b_{2}}$ solving eqn. (43) with $q_{3}=q_{b_{3}}$.

The interpolation factor $\alpha$ is simply computed as

$$
\alpha=\sin \left(q_{5}\right) / \sin \left(\hat{q}_{b_{5}}\right) \simeq q_{5} / \hat{q}_{b_{5}} .
$$

The algorithm has been implemented developing FORTRAN subroutines to be linked to the library of the CYPROS simulation package; the host computer was a $80386 / 80387(24 \mathrm{MHz})$ machine. Although a high-level language and a general-purpose simulation package were used, the available hardware allowed real-time runnings at $15 \mathrm{~ms}$ sampling time for the Euler integration method. In the following, however, results obtained with a sampling time of $1 \mathrm{~ms}$ will be presented.

As a first example, a task space trajectory along which the wrist singularity occurs is given (Figs. 3-8). It consists of a straight line (of $0.72 \mathrm{~m}$ ) to be described with fixed orientation in $3 \mathrm{~s}$ following a trapezoidal profile velocity law $(1 \mathrm{~s}$ of cruise time is assigned). In Figs. 3 and 4 the joint angle histories are depicted; it can be verified that the singularity (i.e. $q_{5}=0$ ) occurs at $t \simeq 1.5 \mathrm{~s}$. Figs. 5 and 6 show the joint velocity histories; the effectiveness of the interpolation can be appreciated by looking at the smooth and continuous behaviour of $\dot{q}_{4}$ and $\dot{q}_{6}$ curves around the singularity which are instead expected to peak close to the singular configuration. Finally, the task-space errors are reported, namely linear and angular velocity errors (Fig. 7) and position and orientation errors (Fig. 8). Remarkably, the magnitude of the velocity transformation errors in the singular region is comparable to that outside the region - where the exact velocity solution is used - which is inherently due to the discrete implementation of the algorithm. This result is even more interesting if related to the smooth joint velocity histories obtained. 


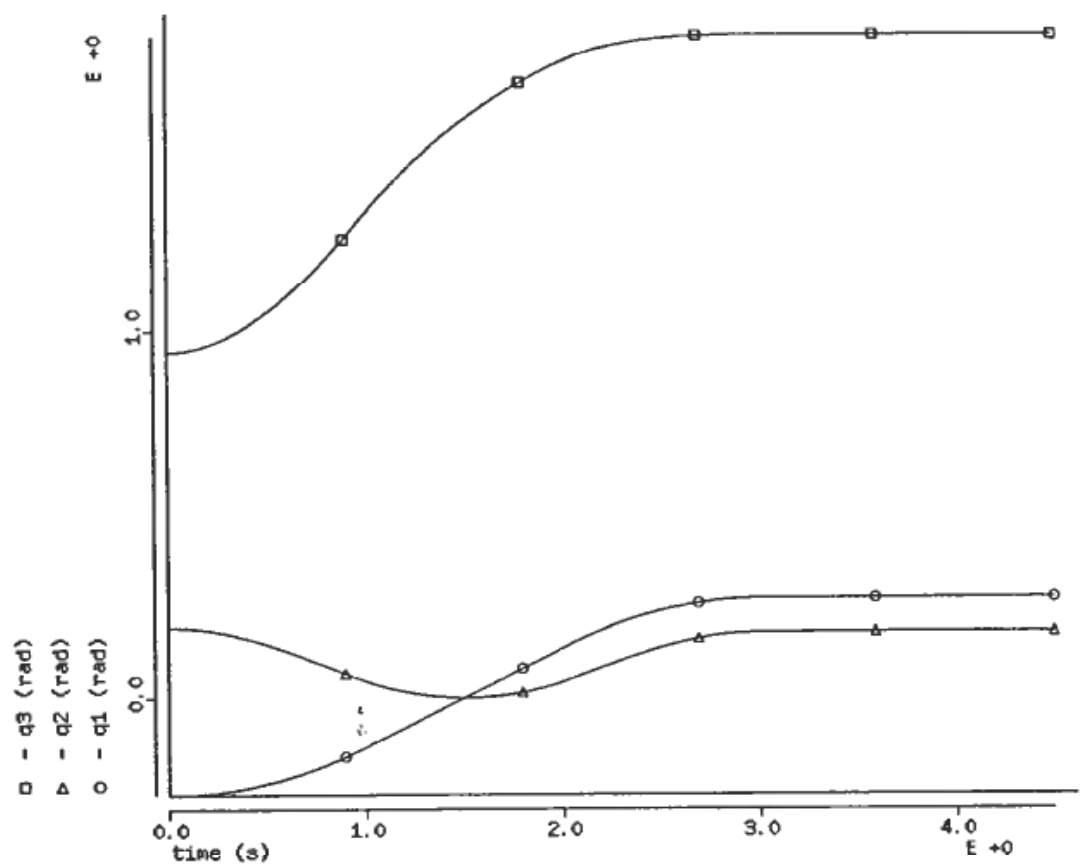

Figure 3. Joint-angle 1-3 histories.

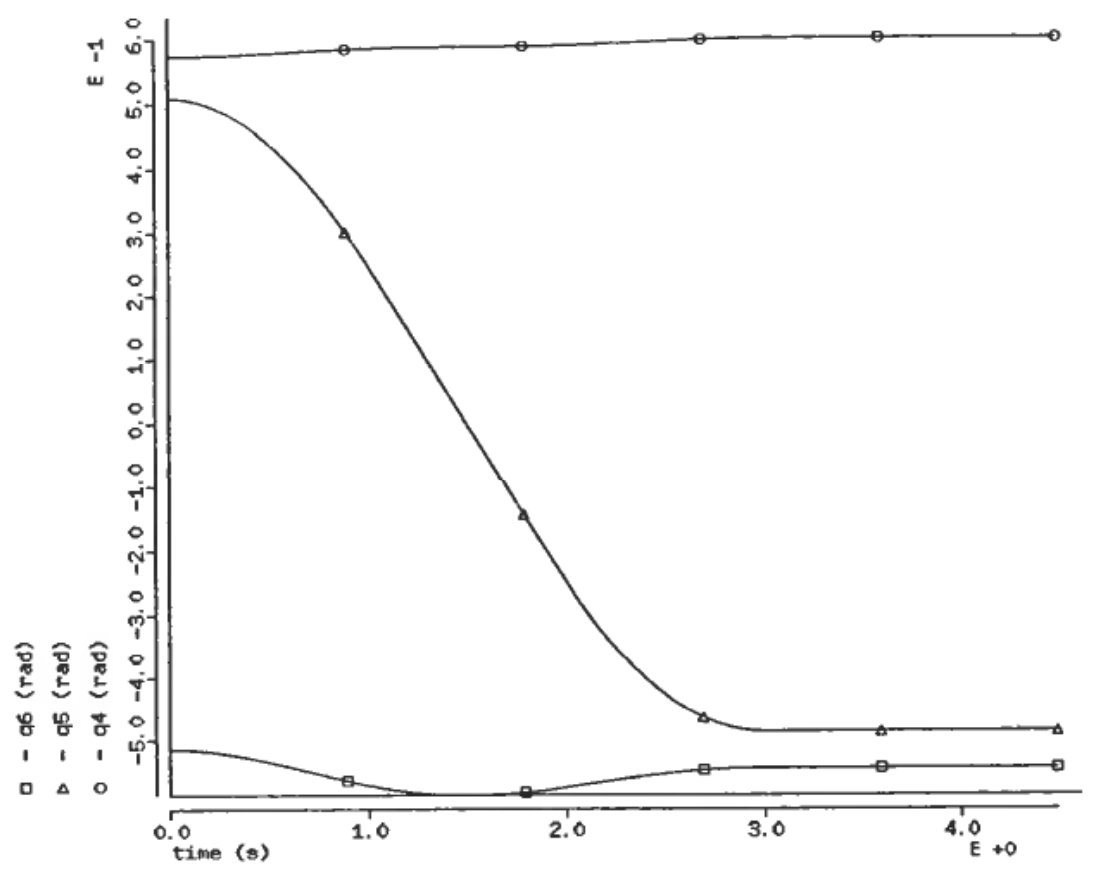

Figure 4. Joint angle 4-6 histories. 


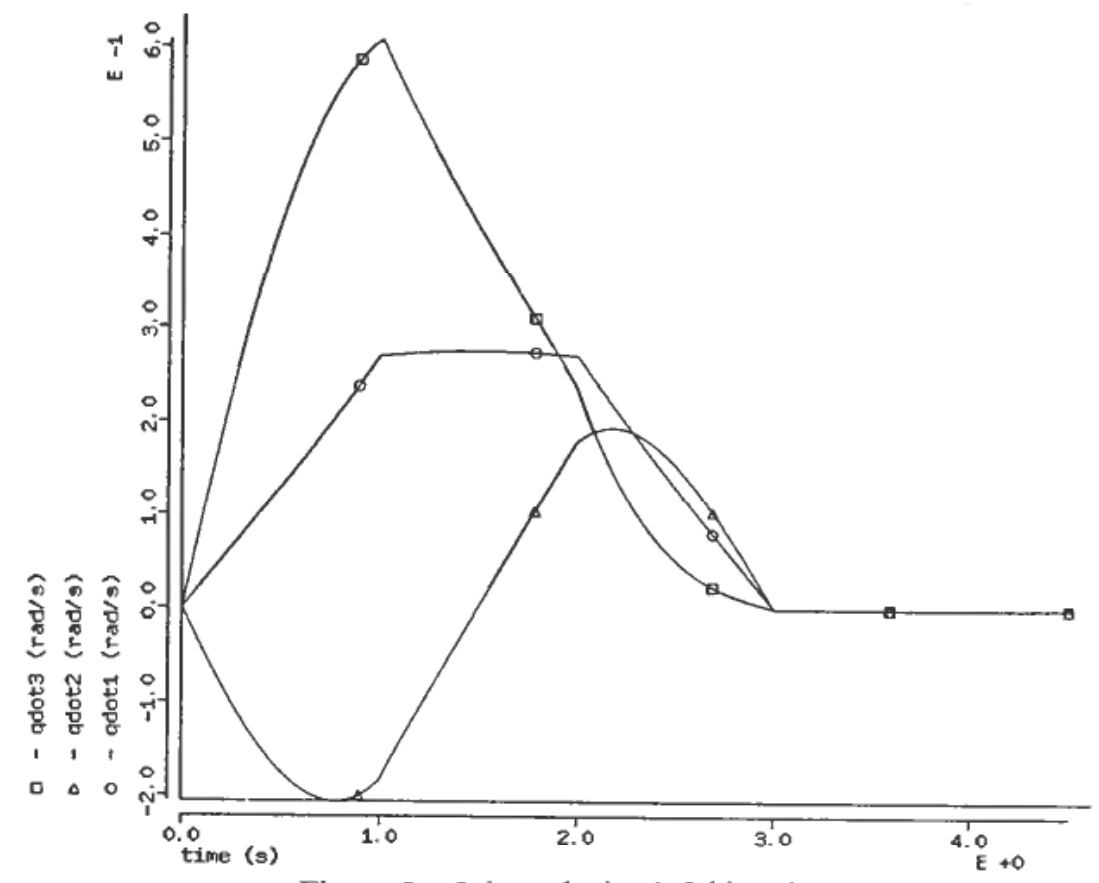

Figure 5. Joint velocity 1-3 histories.

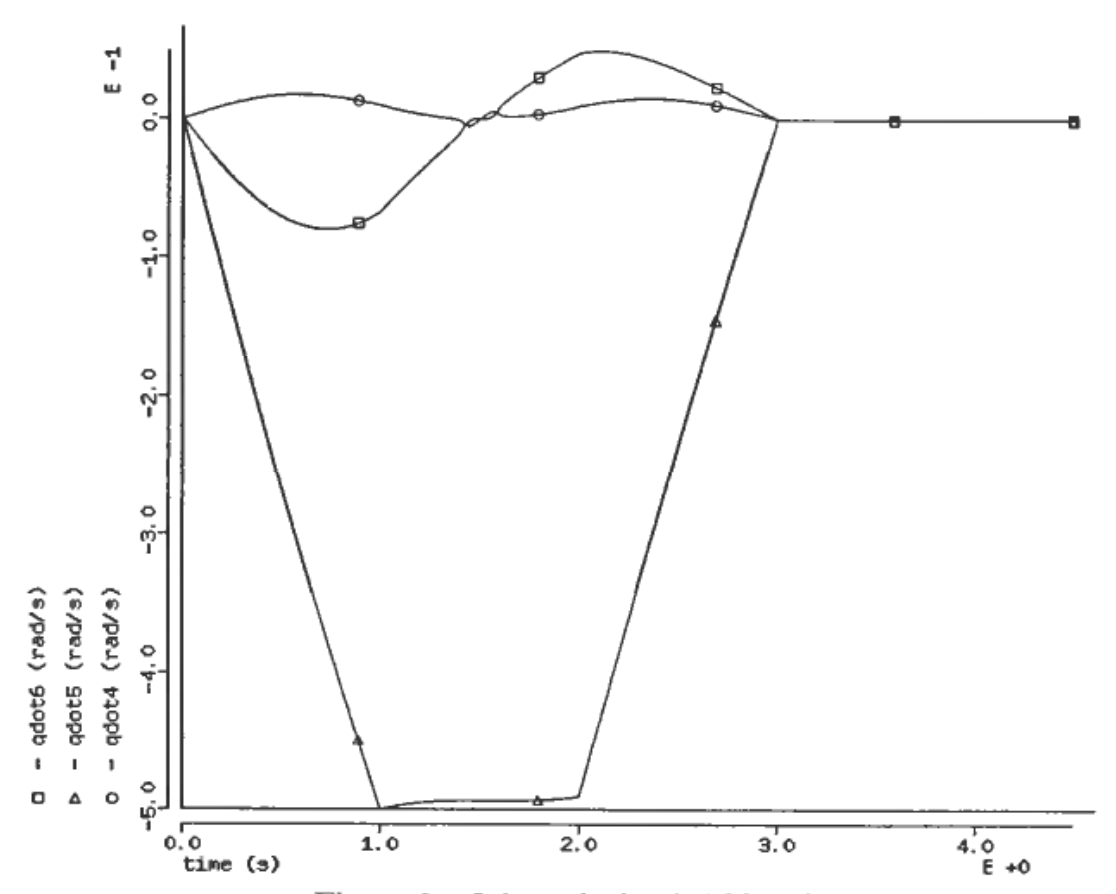

Figure 6. Joint velocity $4-6$ histories. 


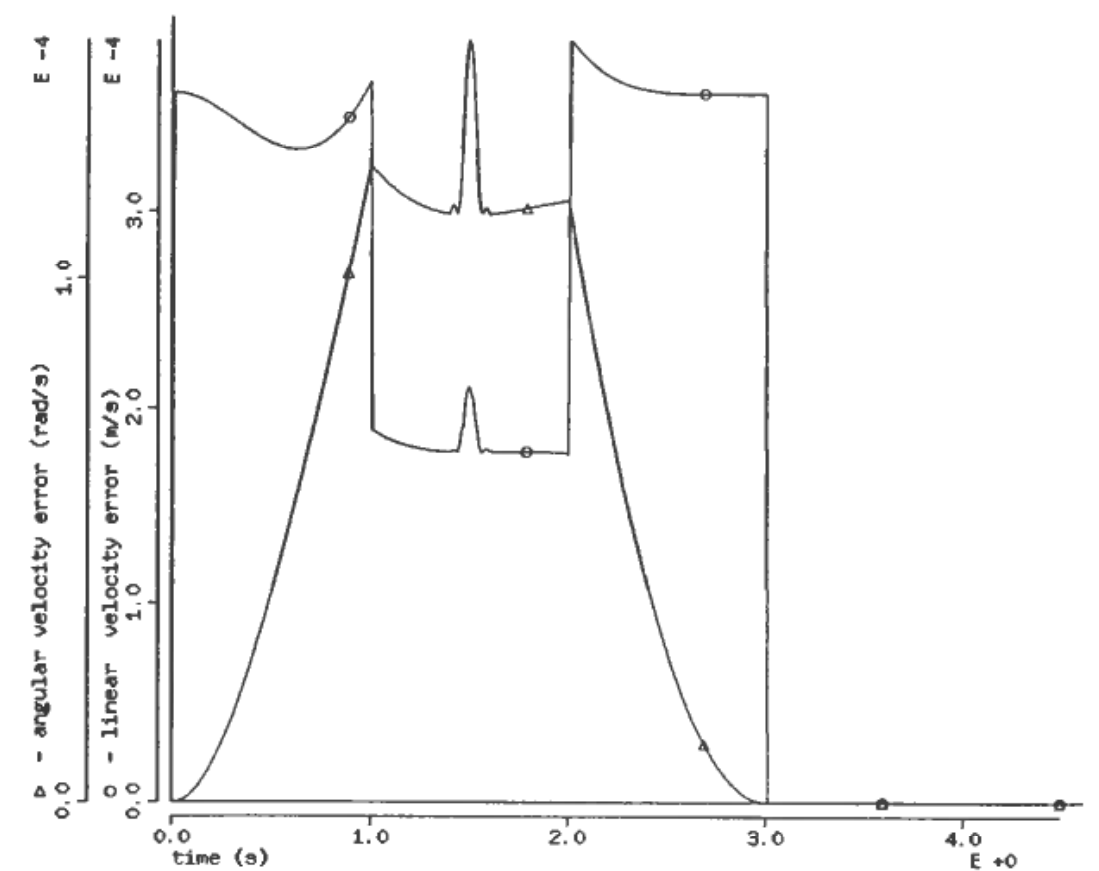

Figure 7. Linear and angular velocity error histories.

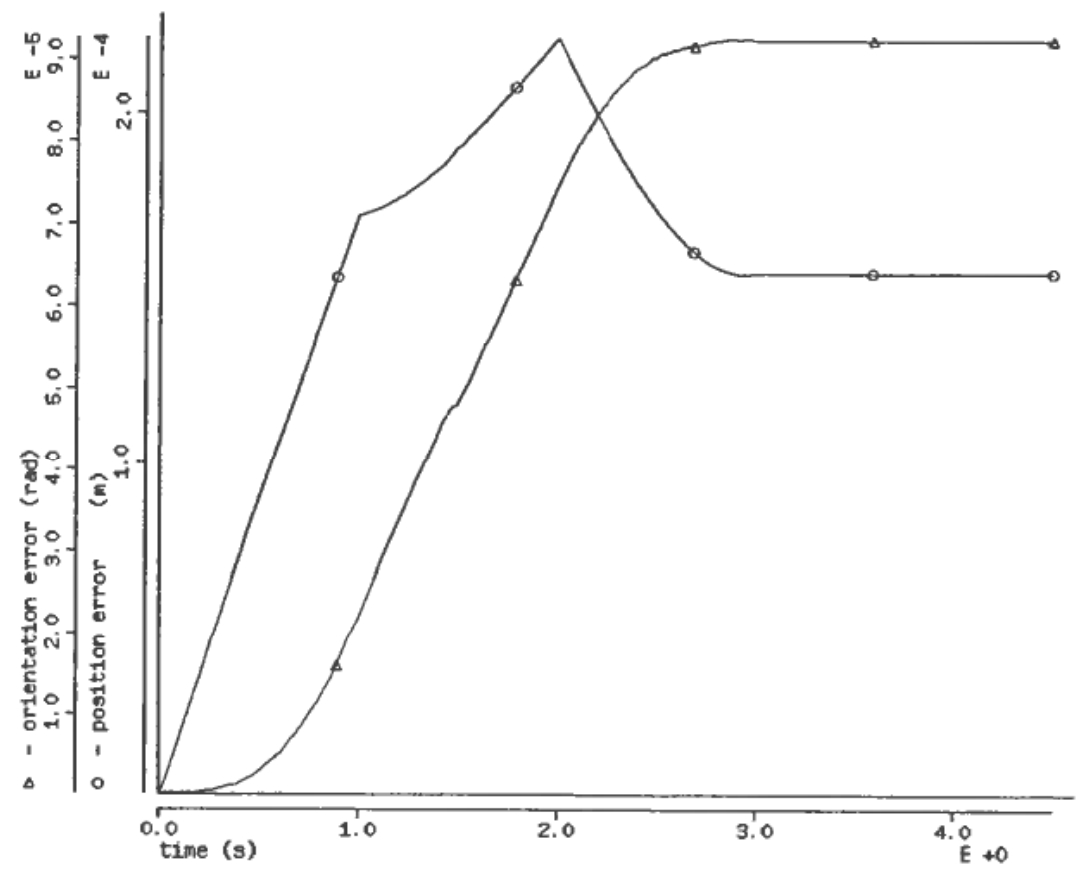

Figure 8. Position and orientation error histories. 


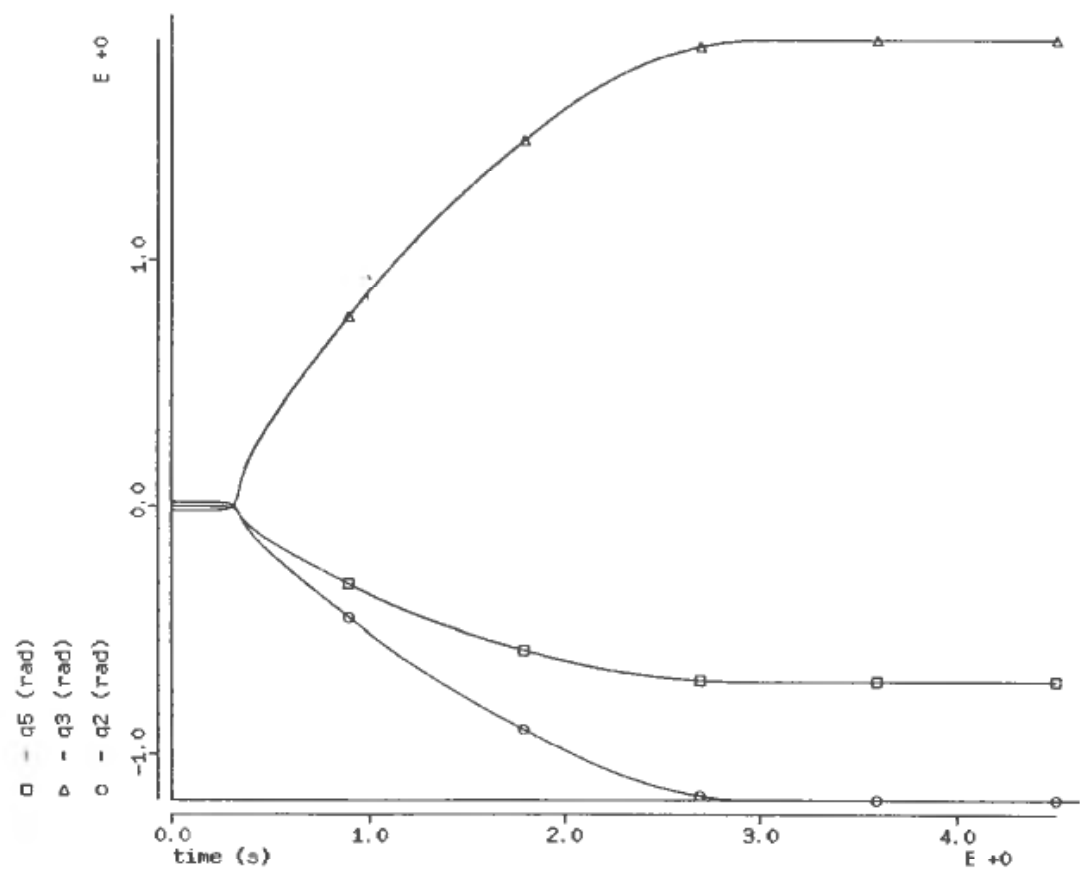

Figure 9. Joint angle histories.

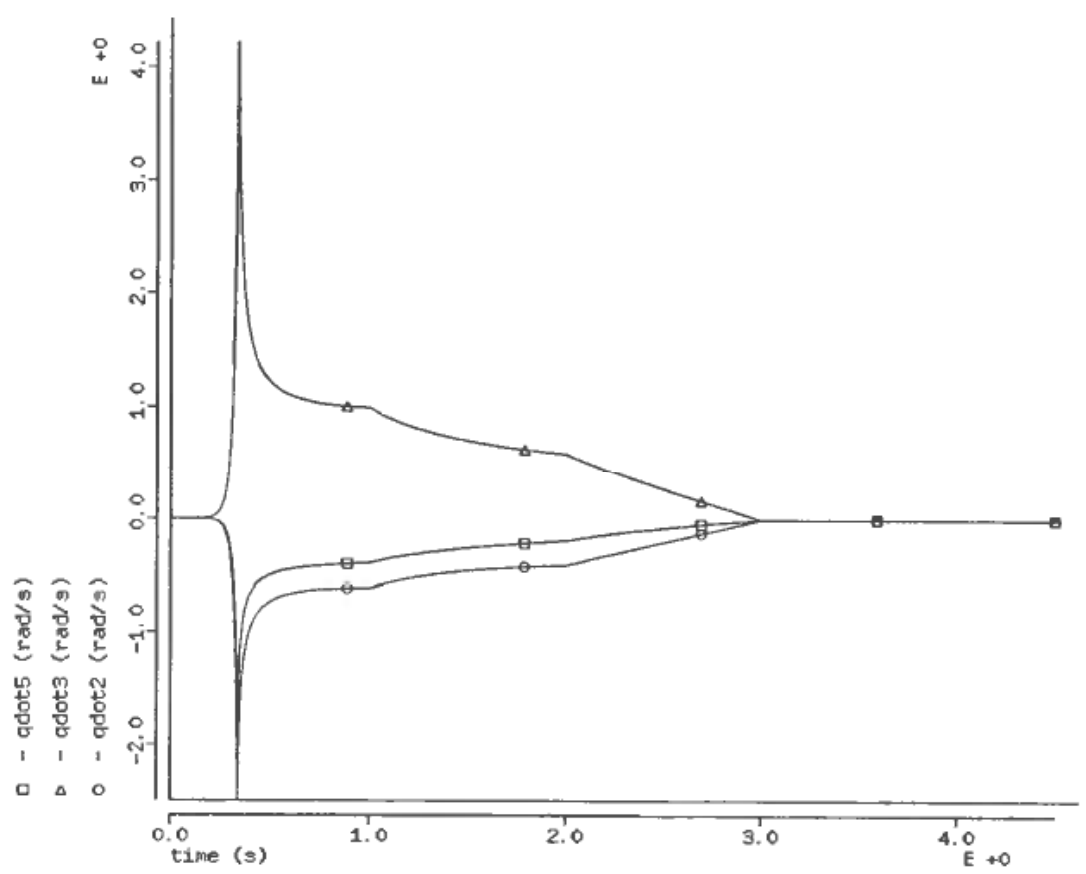

Figure 10. Joint velocity histories. 


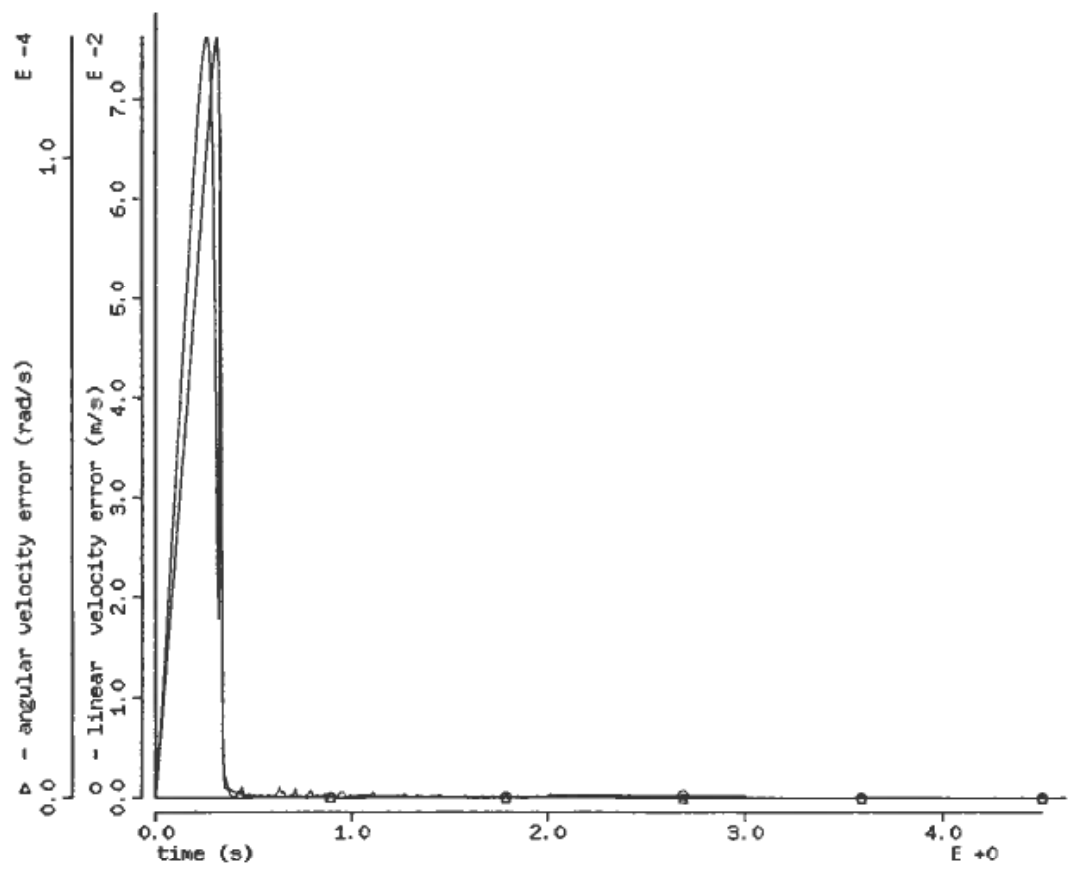

Figure 11. Linear and angular velocity error histories.

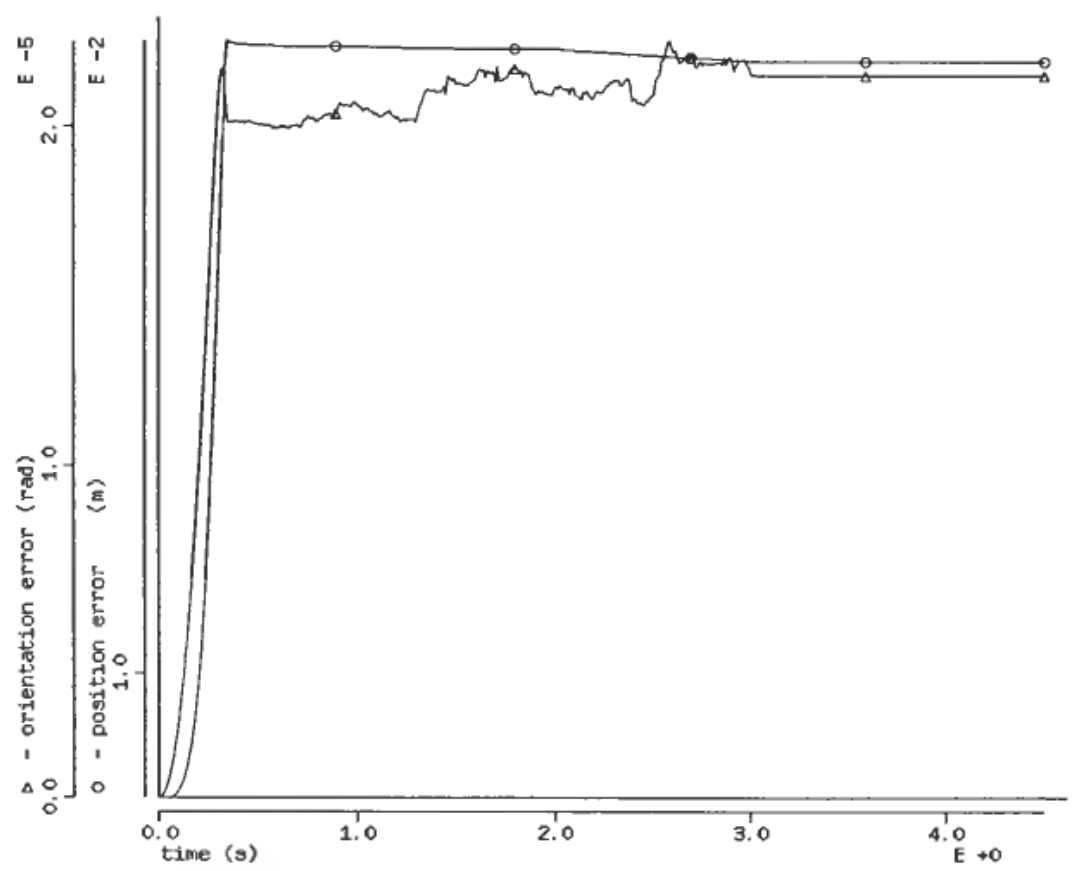

Figure 12. Position and orientation error histories. 
A challenging test for the scheme is constituted by a trajectory which requires to be entirely described with the manipulator in singularity (Figs. 9-12). This is the case of a straight-line trajectory lying on the axis of the robot shoulder; in particular, the manipulator is gradually folded while keeping constant hand orientation. Notice that the starting configuration chosen implies that all three singularities occur. The adopted solution gives no motion to joints 1,4 , and 6 as it enjoys the minimum-norm property; the corresponding curves are therefore omitted. During the earliest stage of the trajectory the manipulator is almost upright; in this phase the commanded motion is not feasible and only the wrist interpolation factor is used. This results in slow arm motion and large task-space velocity errors. As the manipulator leaves the elbow singularity, the commanded motion becomes feasible and joint velocities are increased; subsequently, the wrist singularity is left too. This happens quite suddenly because no elbow interpolation is used. Throughout the transition, peaks on the joint velocity histories are thus experienced. The latest part of the trajectory is properly fulfilled despite the remaining shoulder singularity, as the commanded motion has become feasible.

Further improvement of the tracking performance can obviously be gained if the proposed velocity transformation is integrated in a closed-loop inverse kinematic scheme, thus allowing compensation of the tracking error. In this case, however, larger joint velocities may be required.

\section{Conclusion}

A singularity handling technique for six-joint manipulators has been presented. The inverse kinematic solution is exact in all feasible directions, while an approximation is used in the dependent directions. In the case of a single singularity the solution is continuous, while small discontinuities occur at multiple singularities. The method was demonstrated for the MANUTEC_R3 manipulator, and very good results were obtained with a trajectory through the wrist singularity. The method can be easily extended to other types of manipulators.

\section{ACKNOWIFDGMENT}

This work was done at the German Aerospace Research Establishment (DLR) in Oberpfaffenhofen. The authors would like to thank Dr. G. Hirzinger for his support and encouragement. S. Chiaverini's stay at DLR was partially supported by the NATO Science Programme (Special Programme on Sensory System for Robotic Control) under grant n. 861/88 and by the Ministero della Pubblica Istruzione. O. Egeland's stay at DLR was partly financed by the Royal Norwegian Council for Scientific and Industrial Research.

\section{REFERENCES}

Aboaf, E. W. and Paul, R. P. (1987). Living with the singularity of robot wrists, 1987 IEEE International Conference on Robotics and Automation, Mar.-Apr. 1987. Raleigh, NC, pp. 1713-1717.

Baillieul, J., Hollerbach, J. M. and Brockett, R. W. (1984). Programming and control of kinematically redundant manipulators, 23rd IEEE Conference on Decision and Control, Dec. 1984. Las Vegas, NV, pp. 768-774. 
Balestrino, A., De Maria, G. and SciavicCo, L. (1984). Robust control of robotic manipulators, Preprints of the 9th IFAC World Congress, vol. 6, pp. 80-85.

BEN-ISRAEL, A. and Greville, T. N. E. (1974). Generalized inverses: Theory and applications (New York, Wiley).

Chiacchio, P. and Siciliano, B. (1988). Achieving singularity robustness: an inverse kinematic solution algorithm for robot control, IEE Control Engineering Series 36-Robot Control: Theory and Applications, K. Warwick and A. Pugh Eds. (Hitchin: Peter Peregrinus Ltd.), pp. $149-156$.

Chiaverini, S. and Egeland, O. (1990). A solution to the singularity problem for six-joint manipulators, 1990 IEEE International Conference on Robotics and Automation, May 1990. Cincinnati, OH, pp. 644-649.

DAS, H., Slotine, J.-J. E. and SHERIDAN, T. B. (1988). Inverse kinematic algorithms for redundant systems, 1988 IEEE International Conference on Robotics and Automation, Apr. 1988. Philadelphia, PA, pp. 43-48.

EGELAND, O. (1987). Task-space tracking with redundant manipulators, IEEE Journal of Robotics and Automation, 3, 471-475.

EGELAND, O. (1989). Dynamic coordination in a manipulator with 7 joints, 1989 IEEE International Conference on Robotics and Automation, May 198\%. Scottsdale, AZ, pp. 125-130.

Hollerbach, J. M. (1984). Optimum kinematic design for a seven degree of freedom manipulator, Preprints of the 2nd International Symposium of Robotics Research, pp. 349-356.

Кнатів, О. (1987). A unified approach for motion and force control of robot manipulators: The operational space formulation, IEEE Journal of Robotics and Automation, 3, 43-53.

MAYNE, D. Q. (1969). On the calculation of pseudoinverses, IEEE Transactions on Automatic Control, 204-205.

Nakamura, Y. and Hanafusa, H. (1986). Inverse kinematic solutions with singularity robustness for robot manipulator control. Transactions of the ASME Journal of Dynamic Systems, Measurement, and Control, 108, 163-171.

Nakamura, Y., Hanafusa, H. and Yoshikawa, T. (1987). Task-priority based redundancy control of robot manipulators, International Journal of Robotics Research, 6 (2), 3-15.

SCIAvicCO, L. and Siciliano, B. (1988). A solution algorithm to the inverse kinematic problem for redundant manipulators, IEEE Journal of Robotics and Automation, 4, 403-410.

TAYLOR, R. H. (1979). Planning and execution of straight-line manipulator trajectories, IBM Journal of Research and Developments, 23, 424-436.

WAMPLER II, C. W. (1986). Manipulator inverse kinematic solutions based on vector formulations and damped least-squares method, IEEE Transactions on Systems, Man, and Cybernetics, 16, 93-101.

WAMPLER II, C. W. (1987). Inverse kinematic functions for redundant manipulators, 1987 IEEE International Conference on Robotics and Automation, Mar.-Apr. 1987, Raleigh, NC, pp. $610-617$.

WhitNEY, D. E. (1972). The mathematics of coordinated control of prosthetic arms and manipulators, Transactions of the ASME Journal of Dynamic Systems, Measurement, and Control, 94, 303-309.

Wolovich, W. A. and Elliotr, H. (1984). A computational technique for inverse kinematics, 23rd IEEE Conference on Decision and Control, Dec. 1984. Las Vegas, NV, pp. 1359-1363.

ZADEH, L. and Desoer, C. A. (1963). Linear System Theory, (New York, McGraw-Hill), p. 577. 